\title{
ELM induced tungsten melting and its impact on tokamak operation
}

\author{
J.W. Coenen ${ }^{\mathrm{a}, \mathrm{b}}$, G.Arnoux ${ }^{\mathrm{a}, \mathrm{c}}$, B.Bazyleva ${ }^{\mathrm{a}, \mathrm{e}}$, G.F.Matthews ${ }^{\mathrm{a}, \mathrm{c}}$, S.Jachmich ${ }^{\mathrm{a}, \mathrm{d}}$, I.Balboa ${ }^{\mathrm{a}, \mathrm{c}}$, M.Clever $^{\mathrm{a}, \mathrm{b}}$, R.Dejarnac ${ }^{\mathrm{a}, \mathrm{f}}$, I.Coffey $^{\mathrm{a}, \mathrm{g}}$, \\ Y.Corre $^{\mathrm{a}, \mathrm{h}}$, S.Devaux ${ }^{\mathrm{a}, \mathrm{c}}$, L.Frassinetti ${ }^{\mathrm{a}, \mathrm{i}}$, E.Gauthier ${ }^{\mathrm{a}, \mathrm{h}}$, J.Horacek ${ }^{\mathrm{a}, \mathrm{f}}$, M.Knaup $^{\mathrm{a}, \mathrm{b}}$, M.Komm $^{\mathrm{a}, \mathrm{f}}$, K.Krieger $^{\mathrm{a}, \mathrm{j}}$, S.Marsen $^{\mathrm{a}, \mathrm{k}}$,

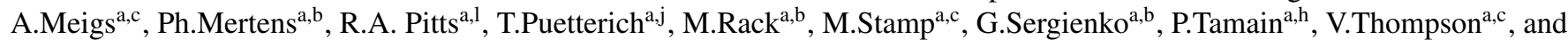 \\ JET-EFDA Contributors ${ }^{\mathrm{m}}$ \\ ${ }^{a} J E T-E F D A$, Culham Science Centre, Abingdon OX14 3DB, UK \\ ${ }^{b}$ Forschungszentrum Jülich GmbH, Institut fuer Energie und Klimaforschung - Plasmaphysik, Juelich, Germany \\ ${ }^{c}$ Culham Centre for Fusion Energy, Abingdon \\ ${ }^{d}$ Laboratory for Plasma Physics, Ecole Royale Militaire/Koninklijke Militaire School \\ ${ }^{e}$ Karlsruhe Institute of Technology, P.O.Box 3640, D-76021 Karlsruhe, Germany \\ ${ }^{f}$ Institute of Plasma Physics AS CR, Za Slovankou 3, 18221 Praha 8, Czech Republic \\ ${ }^{g}$ Astrophysics Research Centre, School of Mathematics and Physics, Queen's Univ. Belfast, UK \\ ${ }^{h}$ CEA, IRFM, F-13108 Saint-Paul-lez-Durance, France \\ ${ }^{i}$ Division of Fusion Plasma Physics, KTH, SE-10044 Stockholm, Sweden \\ ${ }^{j}$ Max-Planck-Institut für Plasmaphysik, 85748 Garching, Germany \\ ${ }^{k}$ Max-Planck-Institut für Plasmaphysik, Teilinsitut Greifswald, D-17491 Greifswald, Germany \\ ${ }^{l}$ ITER Organization, Route de Vinon sur Verdon, 13115 Saint-Paul-lez-Durance, France \\ ${ }^{m}$ (See App. of F.Romanelli et al., Proc. of the 24th IAEA Fusion Energy Conf. 2012, San Diego, USA)
}

\begin{abstract}
In JET-ILW dedicated melt exposures were performed using a sequence of 3MA/2.9T H-Mode JET pulses with an input power of $P_{I N}=23 \mathrm{MW}$, a stored energy of $\sim 6 \mathrm{MJ}$ and regular type I ELMs at $\Delta W_{E L M}=0.3 \mathrm{MJ}$ and $f_{E L M} \sim 30 \mathrm{~Hz}$. In order to assess the risk of starting ITER operations with a full $\mathrm{W}$ divertor, one of the task was to measure the consequences of $\mathrm{W}$ transients melting due to ELMs. JET is the only tokamak able to produce transients / ELMs large enough ( $>300 \mathrm{~kJ}$ per ELM) to facilitate melting of tungsten. Such ELMs are comparable to mitigated ELMs expected in ITER. By moving the outer strike point (OSP) onto a dedicated leading edge the base temperature was raised within $\sim 1 s$ to allow transient ELM-driven melting during the subsequent $0.5 \mathrm{~s}$. Almost $1 \mathrm{~mm}\left(\sim 6 \mathrm{~mm}^{3}\right)$ of $\mathrm{W}$ was moved by $\sim 150$ ELMs within 5 subsequent discharges. Significant material losses in terms of ejections into the plasma were not observed. There is indirect evidence that some small droplets $(\sim 80 \mu m)$ were ejected. The impact on the main plasma parameters is minor and no disruptions occurred. The W-melt gradually moved along the lamella edge towards the high field side, driven by $j \times B$ forces. The evaporation rate determined is 100 times less than expected from steady state melting and thus only consistent with transient melting during individual ELMs. IR data, spectroscopy, as well as melt modeling point to transient melting. Although the type of damage studied in these JET experiments is unlikely to be experienced in ITER, the results do strongly support the design strategy to avoid exposed edges in the ITER divertor. The JET experiments required a surface at normal incidence and considerable pre-heating to produce tungsten melting. They provide unique experimental evidence for the absence of significant melt splashing at events resembling mitigated ELMs on ITER and establish a unique experimental benchmark for the simulations being used to study transient shallow melting on ITER W divertor PFUs.
\end{abstract}

Keywords: PSI-21:Tungsten, Melting, ELM , ILW

PACS: 52.55.Rk, 52.40.Hf, 52.55.Fa, 52.25.Vy

\section{Introduction}

Tungsten (W) is among the main candidate-plasma facing ${ }_{13}$ components (PFC) for a fusion reactor and will be exclusively ${ }_{14}$ used in the ITER divertor. Melting is one of the major risks as- 15 sociated with the material and so PFCs in tokamaks like JET or ${ }_{16}$ ITER are designed in such a way that leading edges and hence ${ }_{17}$ excessive plasma heat load $\left(q_{\|}\right)$are avoided. It was shown $[1,2]_{18}$ that deep W melting can cause severe damage to components ${ }_{19}$ and can degrade plasma performance [3]. The high stored en- 20 ergies of which ITER will be capable means that even with all ${ }_{21}$

Email address: j.w.coenen@fz-juelich.de (J.W. Coenen)

URL: fz-juelich.de/IEK/IEK-4 (J.W. Coenen)
PFC edges protected, shallow surface melting can still occur under disruption and ELM transients. In order to assess the risk of starting ITER operations with a full $\mathrm{W}$ divertor, one of the tasks was to measure the consequences of $\mathrm{W}$ transients melting due to ELMs. JET is the only tokamak able to produce transients / ELMs large enough (> $300 \mathrm{~kJ}$ per ELM) to facilitate melting of W. Such ELMs are comparable to mitigated ELMs expected in ITER [4]. A dedicated misaligned element (lamella) has thus been installed in one part of the bulk W outer divertor, using a tapered exposed edge $(0.25-2.5 \mathrm{~mm})$ permitting exposure of a varying degree of misalignment to the full parallel heat flux $\left(q_{\|}\right)$. The experiment has required dedicated studies on thermal response and significant effort in the interpretation of the corresponding diagnostics. In this contribution 
the general overview of the experimental outcome including material damage evolution, material losses and plasma impact are discussed including arguments relating to important issue of transient vs. bulk melting. Issues related to the actual $q_{s}$ (heat flux to the edge side surface)and the temperature determination as well as additional modeling results are also presented.

\section{Setup}

As part of the JET-ILW [5] JET was equipped with bulk $\mathrm{W}$ modules [6] for the horizontal outer divertor and $\mathrm{W}$ coated tiles for the inner and vertical faces. The main chamber was equipped mainly with Beryllium (cf. fig. 1). This setup is similar to the final ITER material mix and hence allows direct extrapolation of experimental result. Due to power handling

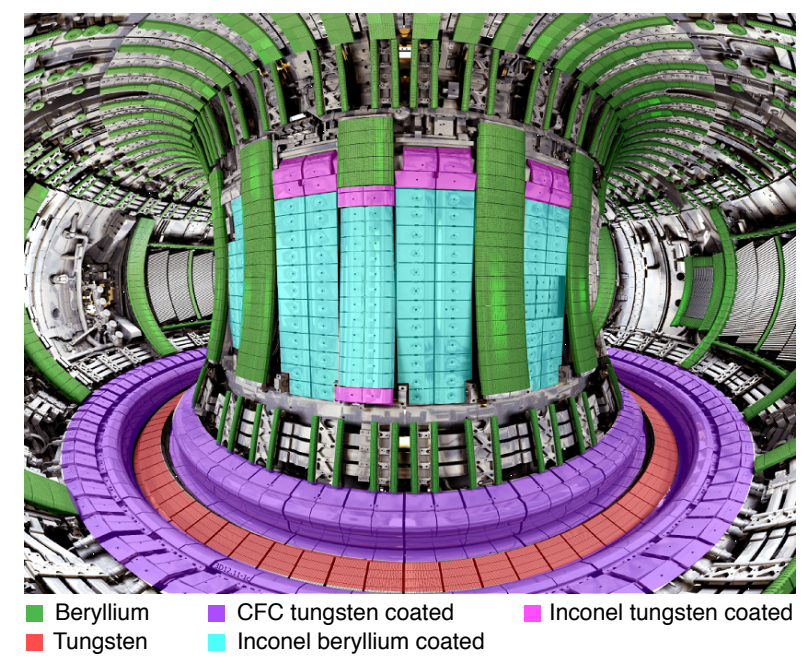

Figure 1: In-vessel view of JET demonstrating the ITER-like Wall material 58 mix. considerations [6] the outer divertor is split up in four so-called ${ }^{59}$ Stacks (A,B,C,D) with A being located closest to the High Field ${ }_{60}$ Side (HFS). Figure 2 displays one of the divertor modules with ${ }_{61}^{60}$ its four stacks and is also displaying the location of required modification with respect to the execution of the described melt ${ }^{62}$ exposures. As the experiment was aimed at ELM induced ${ }_{64}^{63}$

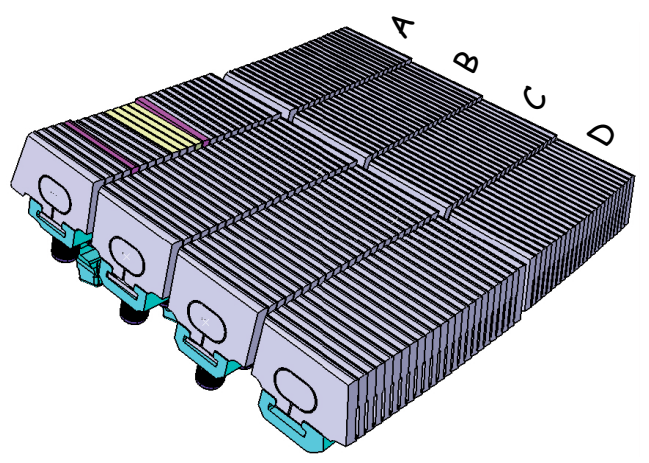

Figure 2: Module of the JET outer divertor depicting the position of the dedi- 78 cated lamella.

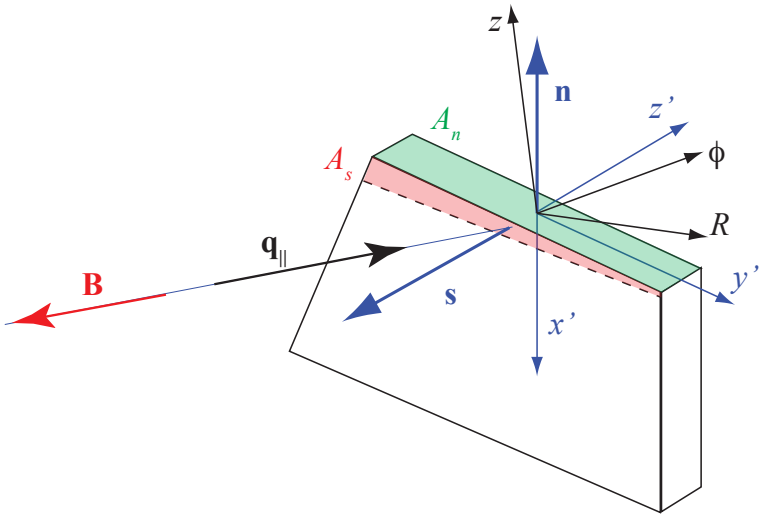

Figure 3: Dedicated lamella.

melting the special lamella was designed to allow significant preheating due to the front surface being exposed to the parallel heat flux (cf. fig. 3)[7]. The exposure to the parallel heat flux is achieved by producing a chamfered leading edge of $0.25-2.5 \mathrm{~mm}$ and also lowering of the 8 lamellas in front of the exposed edge to mitigate potential shadowing (fig. 2). The lamella has a poloidal extent of $5.9 \mathrm{~cm}$ and is $5.5 \mathrm{~mm}$ wide toroidally. On top of the pre-heating ELM induced heat-flux will increase the temperature and hence cause the melting of the exposed surfaces. (cf. sec. 4). Geometrically the relation between $q_{s} \sim q_{\|}$and $q_{n}=q_{\perp}$ should be between 25 and 35 depending on the local field line angle.

$$
\eta_{q}=\frac{q_{s}}{q_{n}}=\frac{\cos \left(\theta_{\perp}+\alpha_{s}\right)}{\sin \left(\theta_{\perp}-\alpha_{s}\right)}
$$

where the field line angle with respect to the target assuming no respective module tilt $\left(\alpha_{s}=0\right)$ is $\theta_{\perp}$.

\section{Diagnostics}

In order to quantitatively interpret the outcome of the experiment and also be able to follow the progress of potential melt damage several additional diagnostics were employed. The toroidal installation position of the special lamella was chosen to allow the existing IR diagnostics $[8,9]$ to be used. The so called K19A and K19B Cameras are of the same type ([10]), $\mathrm{K} 19 \mathrm{~A}$ is covering the area including the special lamella while K19B is focussing on an unchanged reference part of the outer divertor target, as depicted in fig. 4(a). Figure 4(b) shows the actual footprint of the plasma during one of the early exposures of the special lamella, clearly differentiating the temperature rise from its surrounding reference, or flat lamellas.

This top viewing geometry does however mean that the IR camera could only see propagation of the heat pulse into the lamella from the side which creates an issue for interpretation particularly for fast events as discussed below, one pixel of the camera is equivalent to $\approx 1.7 \times 1.7 \mathrm{~mm}$ on the top surface of the lamella.

In order to monitor changes to the installed lamella a highresolution camera was installed (SBIG ST-8300 Monochrome 


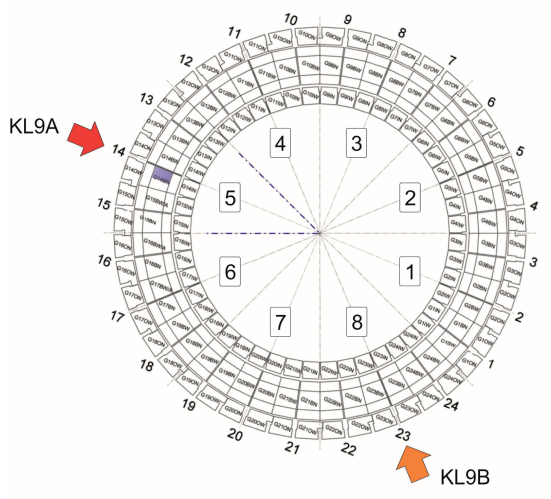

(a) Viewing positions of both IR Kameras

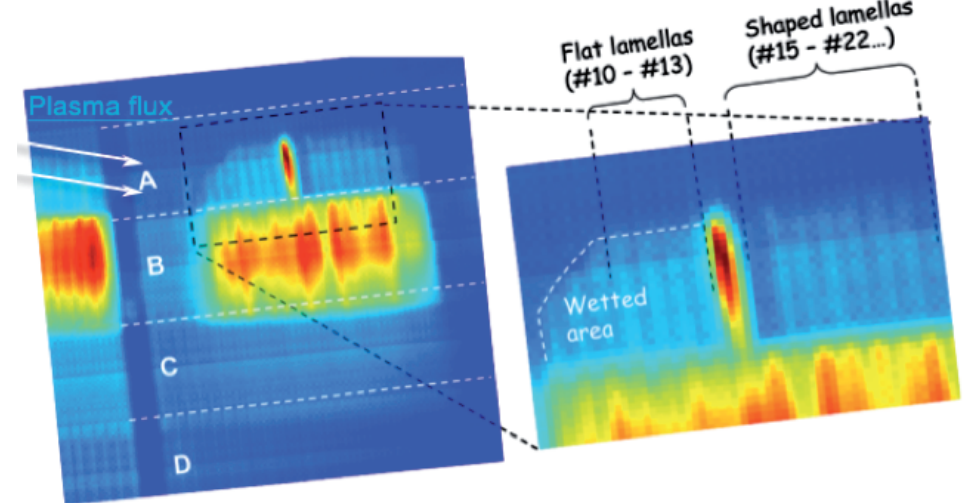

(b) IR impact as seen during Stack A exposure

Figure 4: IR View (KL9A) - heated special Lamella clearly visible (0.5s L-Mode exposure).

[11]). With a resolution of $\sim 100 \mu \mathrm{m}$ one can clearly follow the evolution of the lamella and the surrounding areas as depicted in figure 8(a). No apparent damage to the lamella is observed before the experiments and all edges of the lamellas appear sharp and undamaged.

In order to allow a direct observation of the emitted $\mathrm{W}$ from either evaporation or droplet emission a localized viewing cord was installed. A small observation volume covering the area of the special lamella and part of Stack A allows dedicated measurements. Based on the WI 400.88nm line one can calculate the released amount of $\mathrm{W}$ as demonstrated in $[12,1]$. Experimentally determined photoeffeciencies are used to determine particle fluxes based on line emission. [12] The emission can then be compared to typically evaporation fluxes at given temperatures [13]. From comparing model and experiment it can be determined that vapor-shielding does not play a major role [14], hence no feedback on the W-influx determination is expected from varying plasma parameters. In addition one can expect the photo efficiencies to be constant at high plasma temperatures during ELM impact.

\section{Experiments}

With several preparation pulses the lamella was only carefully and stepwise exposed to longer and higher heat flux to get to the actual experimental conditions.

As prescribed above the configuration for the experiment was chosen as to operate on Stack A. Figure 5 displays the magnetic configuration used for the experiments. Most of the time the strike line was kept on 'Stack B', and only moved to 'Stack A' for short periods of time (0.5-1.5s). The melting occurred during JPN \#84724 (minor) and the sequence from JPN \# 84778-\# 84783 .

Figure 6 displays the parameters of one of the final melt exposures (JPN \#84782). The melt exposures were performed using a sequence of 3MA/2.9T H-Mode JET pulses with an input power of $P_{I N}=23 \mathrm{MW}$ a stored energy of $\sim 6 M J$ and regular type I ELMs at $\Delta W_{E L M}=0.3 \mathrm{MJ}$ and $f_{E L M} \sim 30 \mathrm{~Hz}$. As there is a direct relation between $p_{p e d}$ and the energy fluency [15] to the

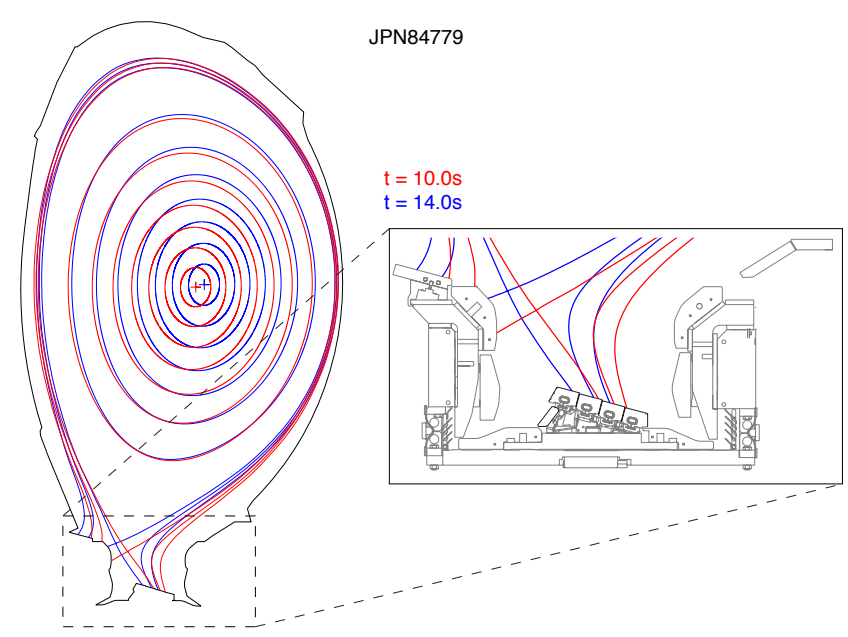

Figure 5: Magnetic configuration during the exposure (blue, Stack A) and before and after the strike-line excursion (red, Stack B).

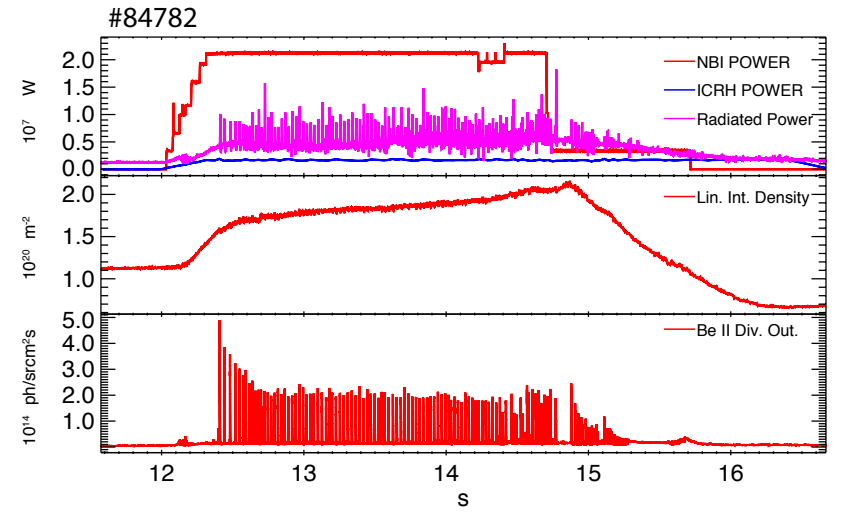

Figure 6: Parameters of JPN \#847782 (bottom) ELM time trace based on BeII Divertor emissions. (mid) Line Integrated Density, (top) Heating Power as well as radiated Power. 
target the pedestal pressure was increased by increasing input power and plasma current up to the condition of the melt exposures. The plasma conditions remained stable even throughout the actual melting (cf. sec 5.4)

In addition to the maximum heat flux to the target during ELMs regular ELMs were required to allow reproducibility of each melt event.

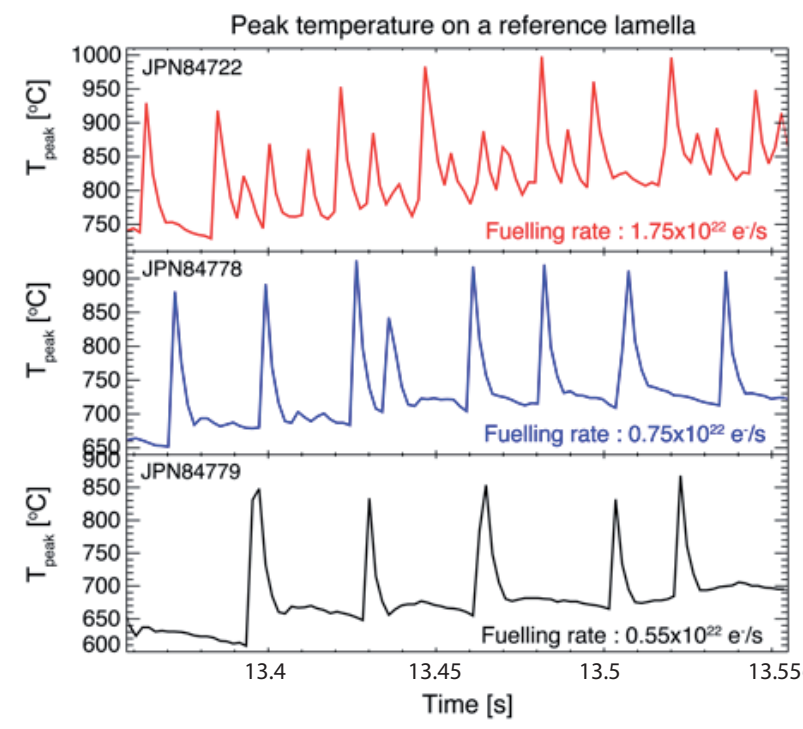

Figure 7: As-Measured temperatures based on IR Thermography for different gas-fuelling conditions.

Figure 7 displays the temperature response of one of the reference lamellas during the final adaption of the gas-fueling rate and hence the related changes in ELM temperature rise and frequency. With decreasing fueling-rate the ELM frequency drops and the temperature rise stabilizes at roughly $200{ }^{\circ} \mathrm{C}$.

\section{Results}

In order to determine the exact outcome of melt experiments it becomes crucial to understand what the actual quantities are which can be determined including their limitations. Available are the top surface measured temperature of the lamella $T(x, y, t)$ $\left[{ }^{\circ} \mathrm{C}\right]$ based on IR thermography as well as visible spectroscopy which allows to determine the $\mathrm{W}$ release rate $W_{\text {eval }}[$ atom/s]. The surface morphology changes can be derived based on high resolution images and compared with the modeled results in terms of melt layer motion and moved amount. All of theses quantities have to form a coherent picture to interpret the melt damage and its consequences for ITER.

\subsection{Melt Damage}

Melting occurred during a series of 7 pulses as shown in fig. 8 , the first of which was \# 84724 where a slight change to the previously straight edge of the lamella can be observed. During the last session beginning with \# 84778 the base temperature was increased by extending the time spent on Stack A to $1.5 \mathrm{~s}$. The observed temperature histories of \# 84778 and subsequent pulses are almost identical (fig. 9) so it seems most likely that

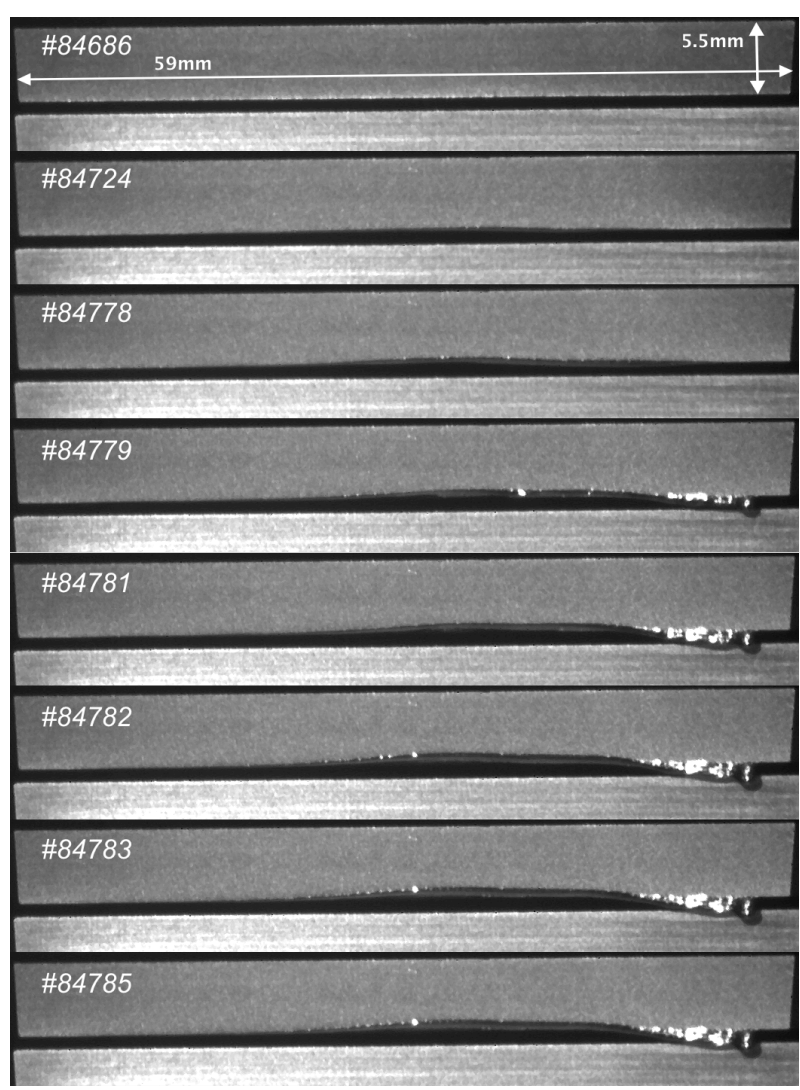

(a) Damage Evolution during the relevant pulses

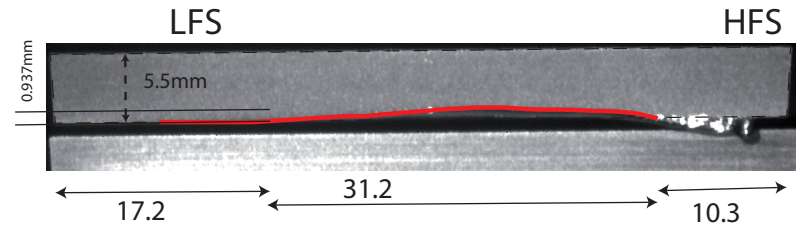

(b) Geometrical considerations incl. damage profile

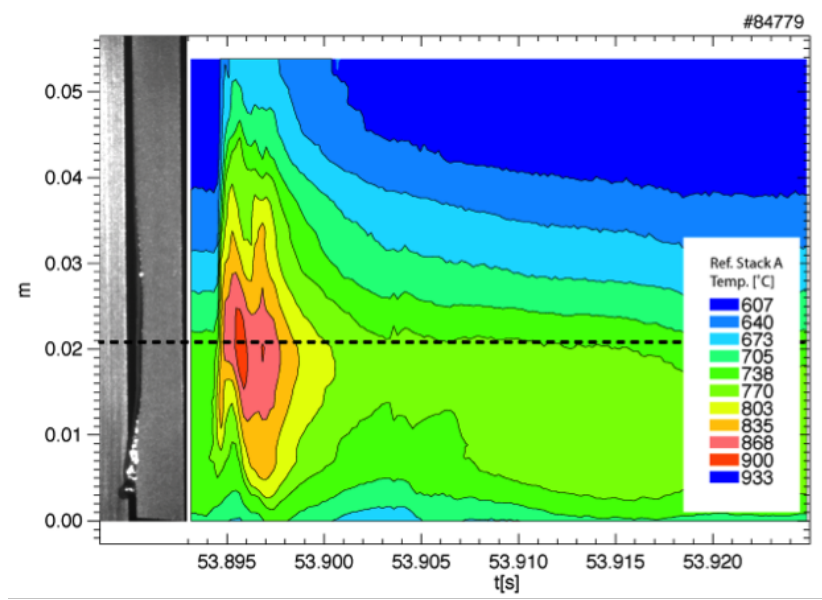

(c) Comparison of inflicted damage and ELM temperature rise

Figure 8: Melt damage inflicted during the melt exposures, as well as geometrical comparisons incl. a temperature footprint from a reference lamella. 
the formation of the large droplet chain in \#84779 is a cumula-182 tive effect rather than the result of a change in the parameters ${ }_{183}$ of the incident plasma. The actual material moved can be es- 184 timated by geometrical arguments from fig. 8(b) and has to 185 be confirmed by post-mortem analysis after the 2014 JET cam-186 paigns. From the estimation one can deduce that $6.2 \mathrm{~mm}^{3}$ were 187 moved which is roughly consistent with the estimated mass of 188 the droplets visible on the HFS of the lamella. There is also 189 clear evidence from these pictures that the droplets move along ${ }_{190}$ the edge, coalesce and grow. Fig. 8(c) shows the damage next ${ }_{191}$ to the ELM temperature rise. Overlaying the temperature mea-192 surement from a reference lamella, one can correlate the visible ${ }_{193}$ damage profile with the peak temperature values. The refer-194 ence lamella is not exposed to the parallel heat flux and has no195 leading edge.

The damage visible is about $200 \mu \mathrm{m}$ per pulse caused by 197 30ELMs per exposure. Each ELM hence removes $\sim 7 \mu m_{198}$ The impact on the material properties can only be determined ${ }_{199}$ once the lamella has been analyzed outside the vessel. From 200 $[16,17,18]$ its is well known that strong grain growth can occur201 which in turns leads to diminished power handling capabilities 202 during transients.

\subsection{Temperature Determination}

A crucial point with respect to the experiments is the temperature evolution of the exposed lamella and its front surface ${ }_{208}$ and hence the actual relation of heat fluxes to the melt behavior and melt layer motion. Due to the complexity of this ${ }_{209}$ part of the analysis a dedicated analysis is given in [14], while ${ }_{210}$ only the main results are given here. Figure 9 displays the as- ${ }_{211}$ measured peak temperature values on both a flat reference $\mathrm{as}_{212}$ well as the special lamella for each of the $1.5 \mathrm{~s}$ long melt expo- ${ }_{213}$ sures (\# 84778-\# 847782). One can clearly see the reproducibil- ${ }_{214}$

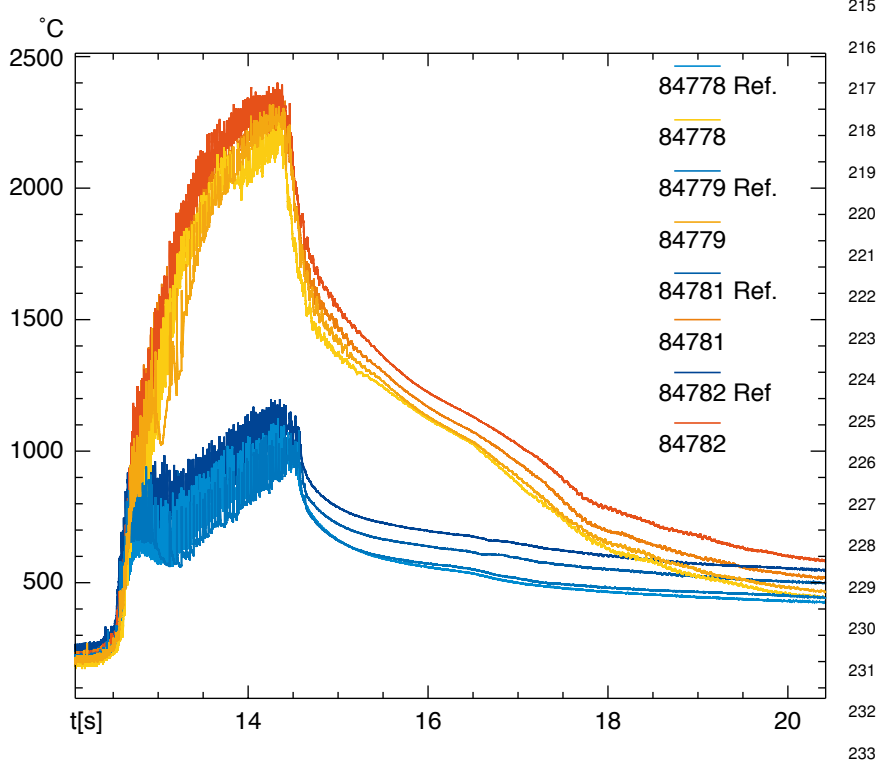

Figure 9: Temperature Evolution for the reference (blue ) as well as the special ${ }^{234}$ lamella (orange-red) but also the differences between the special and the reference lamella. The temperature rise for the special lamella is roughly $\Delta T=2200 \mathrm{~K}$ whith the peak close to $2500^{\circ} \mathrm{C}$ while the reference lamella at most heats up to $1100^{\circ} \mathrm{C}$. Since, due to the limited resolution of the IR diagnostics $(1 \mathrm{px}=1.7 \mathrm{~mm})$, temperature gradients aren't properly reflected in the measurement, temperatures on the leading edge - located within one pixel- are measured lower for both the slow temperature evolution but also the ELM induced temperature rise.(cf. [14]).

On slow timescales the effect is only up to $30 \%$ while during ELMs with much steeper gradients a factor of 3-5 can be determined by modeling the actual thermal diagnostic response (cf. [14]) based on data. This means that the peak temperature of the exposed lamella is much closer to $2800^{\circ} \mathrm{C}$ with an ELM induced temperature rise of close to $1000^{\circ} \mathrm{C}$ on top. Melting during each ELM can hence be achieved.

With the assumptions discussed earlier (fig. 3 , eq. 1) one would expect that the temperature excursion on the special lamella should be close to a factor 25 higher when comparing to the excursion on the reference. This is obviously not the case as seen in figure 9 . Eventhough the direct relation between $\Delta T$ and $\Delta q$ is only observed assuming $1 \mathrm{D}$ heat diffusion one can assume that not the full parallel heat flux is actually reaching the leading edge. This is confirmed by $3 \mathrm{D}$ modeling. The actual melt damage and other results are consistent with the temperature determined hence an explanation for the diminished heat-flux impact is still required.

\subsection{Modeling}

In order to validate the finding on the material damage modeling was employed. The obvious starting point was to use the measured perpendicular heat-fux as input into the 3D thermal model and melt layer dynamic codes [19, 20, 21]. For the details of the modeling and the thermal data interpretation refer to [22].

As seen already from the temperature behavior assuming the geometric heat flux to reach all surfaces as expected will cause unrealistic temperature evolutions. The maximum temperature would rise up to $\mathrm{T}>6000 \mathrm{~K}$ after only a few $100 \mathrm{~ms}$. In addition, the depth of the melt pool would be close to $\mathrm{m}$. In order to match the experimentally observed situation a mitigation factor is applied. The factor is applied to the parallel heat flux reaching the exposed edge and is close to 0.3-0.4 depending on the assumptions. For both H-Mode and L-Mode conditions a mitigation factor is required [14]. With physics understanding improving, factors dependent on exposure conditions may however prove necessary. So far a clear physics picture of the mitigating factor is lacking. Figure 10 shows values for the perpendicular heat-flux, the temperature evolution as-measured and as simulated and a comparison of the modeled and measured $\mathrm{W}$ evaporation-rate. When applying a mitigation factor to the parallel heat flux the temperature evolution begins to match and also the evaporation rate gets close to the measured value. Correlating the time history of melting caused by ELMs of the order of ms and taking into account the time averaged evaporation rate measurement $(100 \mathrm{~ms})$ one can clearly determine that the evaporation rate can only be consistently explained by transient 


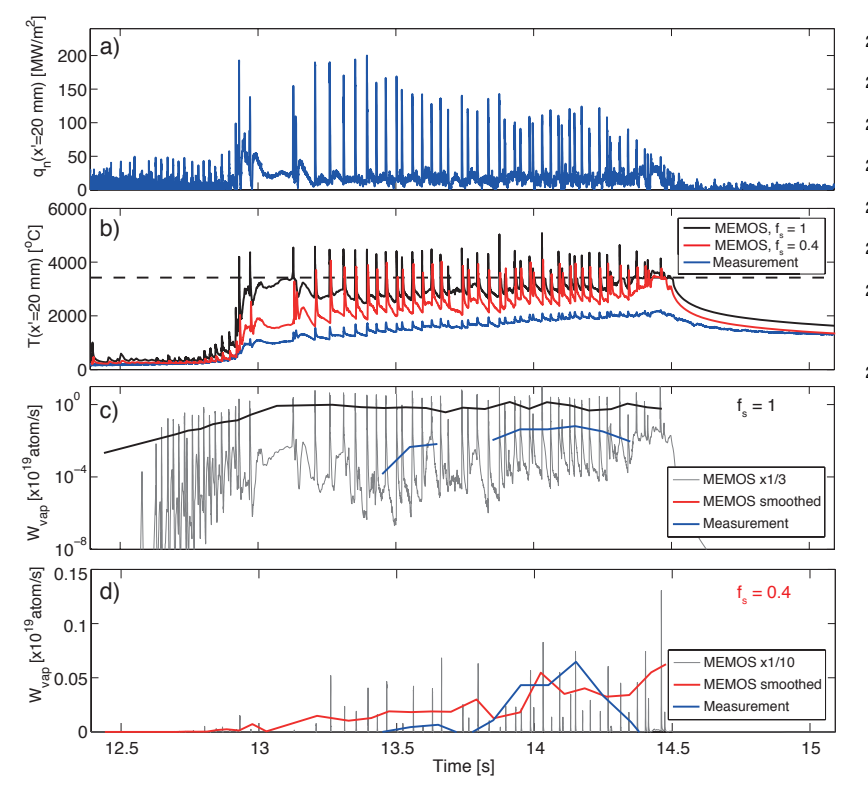

Figure 10: Comparison of experimental data for \#84779 and modeled results for temperature and W-release rate.

melting. Taking into account the mitigating factor and matching the temperature evolution between experiment and modeling one can now estimate the actual melt damage and redistribution based on the model (MEMOS [22]) Figure 11 shows the

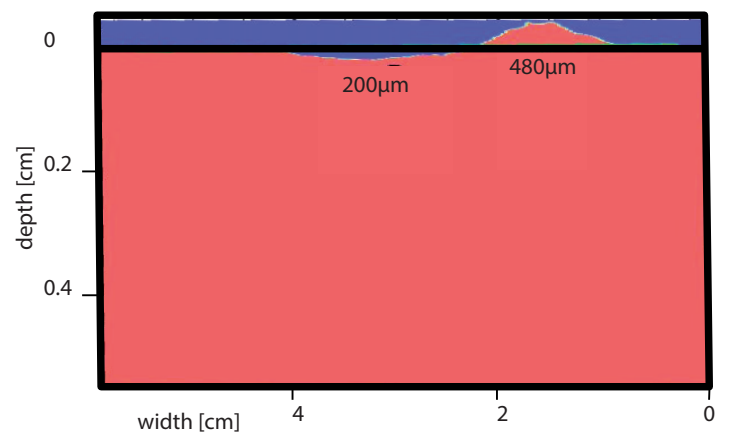

Figure 11: Modelled melt layer re-distribution after one pulse (based on272 \#84779).

melt layer redistribution assuming a mitigating factor of $0.3 \mathrm{as}_{275}$ also used in fig. 10. One exposure is modeled based on the 276 ment with the observed melt damage and redistribution pattern 278 the melt is moving towards the HFS with one pulse roughly $y_{279}$ causing $200 \mu \mathrm{m}$ damage. The dominant forces leading to this 280 redistribution are related to a thermo-electric current driven $\mathrm{jxB}_{281}$ force, as seen from previous melt experiments [19].

In order to explain this mitigating factor so called Larmor-283 radius smoothing was considered $[23,24]$ causing mitigated 284 heat flux to exposed surfaces due to gyration effects related to285 high-energetic particles from ELMs. Taking into account the 286 pedestal profiles during the actual experiments ELMs as ob-287 served during the experiments will show a redistribution of heat288 heat flux data given for JPN \# 84779 (cf. fig. 10). In agree-277

flux along the leading edge but only accounts for only $20-30 \%$ of the observed effect. As the effect is visible during L-Mode as well Gyro-effects cannot be the main contribution.

Hence allowing for a mitigated heat-flux to be taken into account one can match the temperature evolution, the actual melt depth and redistribution pattern but also the evaporation rate as an independently determined quantity.

\subsection{Impact on the plasma}

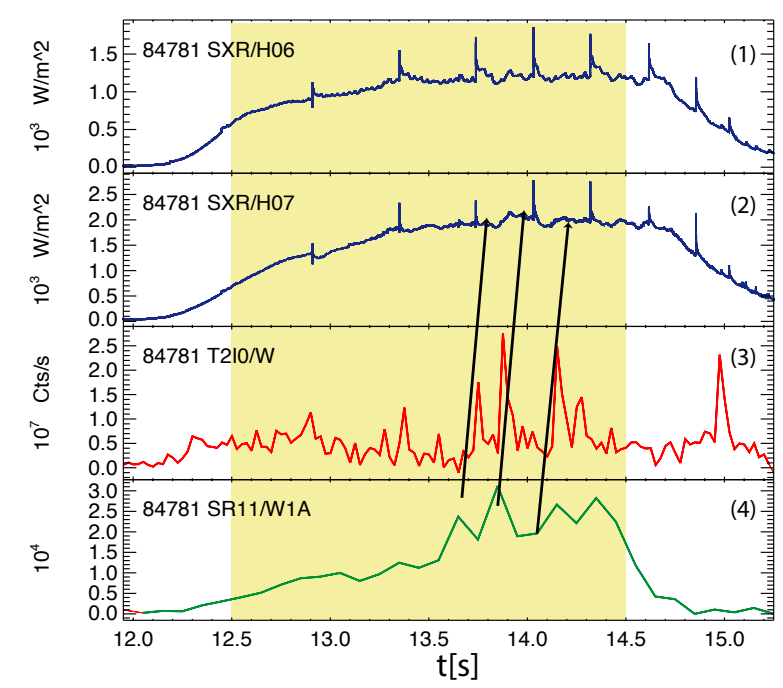

Figure 12: Signals for local as well as core plasma impact regarding the melt tungsten source. (1)(2) Soft Xray , (3) Edge VUV W Signal (4) Local WI (400.9nm)

With respect to the impact on operation two main aspects need to be considered, firstly the W-source originating from evaporation of the strongly heated and molten exposed surface and secondly the potential influence of droplet expelled into the divertor plasma and potential transport of ejected mass into the main plasma. Figure 12 depicts three $\mathrm{W}$ related signals, local WI (400.9) emissions, W-VUV emissions as well as Soft X-Ray signals. Spectroscopic measurements in the VUV were performed with the aid of a set of survey SPRED spectrometers $[25,26]$. Using either routinely a $450 \mathrm{~g} / \mathrm{mm}$ holographic grating in the $10-110 \mathrm{~nm}$ wavelength range employing a horizontal line-of-sight into the plasma or a spectrometer with a $2105 \mathrm{~g} / \mathrm{mm}$ grating recording spectra in the wavelength range below $40 \mathrm{~nm}$ and looking nearly vertically down into the JET divertor. This system is sensitive to tungsten ions in the charge state range $\mathrm{W}_{27+}$ to $\mathrm{W}_{35+}$ which are indicative of the influx of tungsten ions reaching mid-radius of the plasma [27, 28]. The Soft X-Ray Data (SXR) $(\sim 0.8 \mathrm{~nm})$ corresponds to the core plasma [27]. The local source increases with extended exposure duration due to the enhanced surface temperature. Some droplets are ejected (spikes in the WI) which are then subsequently also observed in the VUV emission as well as the main plasma (SXR). Combining the three different signals a sequence of movement from divertor to core plasma can be inferred. 
It can be estimated that the W-concentration due to each droplet increases by $\Delta c_{W} \sim 3 \cdot 10^{-5}$. This corresponds to a fully evaporated W-droplet of $r \sim 80 \mu \mathrm{m}$. The impact on the plasma is minor despite an increase in radiated power. No disruption was caused by any of theses events which in principle are similar to impurity events observed with the ILW [29, 30, 31]. The actual droplet size leaving the surface can not be determined due to the long lifetime of droplets leaving the local observation volume, and potentially passing thourgh divertor and main plasma [1, 32]. There are indication that the apparent size of the droplets is influenced by shielding effects however [33].

\section{Conclusion and Outlook}

The JET ELMs were of a size relevant to mitigated ELMs in ITER. Melting due to ELMs in JET is reproducible and well behaved with of order $200 \mu \mathrm{m}$ per pulse being removed from the exposed edge. Melting by ELMs causes an enhanced W-source including occasional expulsion of small droplets $(80-100 \mu \mathrm{m})$ which do not significantly impact the main plasma. Melt layer motion appears predominantly driven by $\mathrm{JxB}$ forces implying significant current flow during ELMs. The melted material moves predominantly into the private region out of the main heat flux area. The droplets can coalesce and grow creating a disruption risk if they were to be ejected into the main plasma. The physics of $\mathrm{W}$ droplets should be machine size independent whereas screening and resistance to the effects of $\mathrm{W}$ radiation improves with machine size. The results are directly relevant to what would happen in the case of an exposed edge. They also suggest that provided such an event is detected and is not repeated too many times such that large droplets build up, there would be no risk of a disruption in ITER. The limited impact shown in these results clearly favored the start with all W. Given the relatively short time the lamella spent over the melt threshold the material loss rate is high with potential lifetime implications. This supports the need for early detection in ITER coupled with mitigation measures. Obviously ITER has the potential to produce similar damage over the whole area of the strike point. The number of droplets produced could therefore be much larger. To disrupt an ITER plasma several large droplets might be required. The JET results do provide basis for further studies in this area including modeling.

\section{Acknowledgement}

This work, supported by the European Communities under the contract between EURATOM and FZJ, was carried out within the framework of the European Fusion Development Agreement. The views and opinions expressed herein do not necessarily reflect those of the European Commission or of the ITER Organization.

[1] Coenen, J. W. et al. Nuclear Fusion, 51 (2011), 8, 083008.

[2] Krieger, K. et al. Physica Scripta, T145 (2011), 014067.

[3] Lipschultz, B. et al. Nuclear Fusion, 52 (2012), 12, 123002.

[4] Pitts, R. et al. Journal of Nuclear Materials, 415 (2011), 1 SUPPL, -

[5] Matthews, G. F. et al. Physica Scripta, 2007 (2007), T128, 137.

[6] Mertens, P. et al. Journal of Nuclear Materials, 415 (2011), s943-s947.
[7] Mertens, P. et al. Proc. 24th IAEA Fusion Energy Conf.(FEC2012), San Diego, USA, EX/PS-24 (2012).

[8] Arnoux, G. et al. Review of Scientific Instruments, 83 (2012), 10, 10 D727.

[9] Balboa, I. et al. Review of Scientific Instruments, 83 (2012), 10, 10 D530.

[10] Eich, T. g. et al. Journal of Nuclear Materials, 415 (2011), 1 SUPPL, S856-S859. Cited By (since 1996) 16.

[11] STF-8300M - https://www.sbig.com/products/cameras/stf-series/stf/stf$8300 \mathrm{~m} /$.

[12] van Rooij, G. et al. Journal of Nuclear Materials, 438 (2013), S42.

[13] T.Tanabe. Atomic and Plasma-Material Interaction Data for Fusion, 5 (1994), 129.

[14] Arnoux et al., G. PSI 2014 - submitted to Journal of Nuclear Materials, (2014).

[15] Sieglin, B. et al. Plasma Physics and Controlled Fusion, 55 (2013), 12, 124039

[16] Coenen, J. W. et al. Fusion Science And Technology, 61 (2012), 2, 129135.

[17] Coenen, J. W. et al. Physica Scripta, T145 (2011), 014066.

[18] Coenen, J. W. et al. Journal of Nuclear Materials, 415 (2011), 78-82.

[19] Bazylev et al., B. Physica Scripta, T145 (2011), 014054.

[20] Bazylev, B. et al. Fusion Engineering and Design, 83 (2008), 7-9, 1077 1081.

[21] Bazylev, B. et al. Journal of Nuclear Materials, 363-365 (2007), 10111015.

[22] Bazylev et al., B. PSI 2014 - submitted to Journal of Nuclear Materials, (2014).

[23] Dejarnac et al., R. submitted to Nuclear Fusion, (2014).

[24] Dejarnac, R. et al. Journal of Nuclear Materials, 415 (2011), 1, Supplement, S977 - S980. Proceedings of the 19th International Conference on Plasma-Surface Interactions in Controlled Fusion.

[25] Coffey, I. H. and Contributors, R. B. J. E. Review of Scientific Instruments, 75 (2004), 10, 3737-3739.

[26] Wolf, R. C. et al. JET IOP Preprint, (1995). JET-P(95)34.

[27] Puetterich, T. et al. Plasma Physics and Controlled Fusion, 55 (2013), 12, 124036.

[28] Puetterich, T. et al. Plasma Physics and Controlled Fusion, 50 (2008), 8, 085016.

[29] Coenen, J. et al. Nuclear Fusion, 53 (2013), 7, 073043.

[30] Sertoli et al, M. Physica Scripta, 2014 (2013), T159, 014014.

[31] Sertoli et al., M. PSI 2014 - submitted to Journal of Nuclear Materials, (2014).

[32] Krieger, K. et al. Journal of Nuclear Materials, 451 (2011), 211.

[33] Smirnov, R. D. et al. An impact of w dust on the edge plasma in iter. Priv. comm. 